Fakten und Statistiken 2003

\title{
3. Zusammenarbeit mit Ländern Osteuropas und der GUS
}

\section{Monika Egger}

\section{(2) OpenEdition \\ 12 Journals}

Electronic version

URL: http://journals.openedition.org/sjep/255

DOI: 10.4000/sjep.255

ISSN: 1663-9677

Publisher

Institut de hautes études internationales et du développement

\section{Printed version}

Date of publication: 1 avril 2003

Number of pages: $43-48$

ISBN: 2-88247-050-3

ISSN: $1660-5926$

\section{Electronic reference}

Monika Egger, « 3. Zusammenarbeit mit Ländern Osteuropas und der GUS », Schweizerisches Jahrbuch für Entwicklungspolitik [Online], 22-1 | 2003, Online erschienen am: 04 Mai 2010, abgerufen am 10 Dezember 2020. URL : http://journals.openedition.org/sjep/255 ; DOI : https://doi.org/10.4000/sjep. 255

(c) The Graduate Institute 


\section{ZUSAMMENARBEIT MIT LÄNDERN OSTEUROPAS UND DER GUS*}

D IE SCHWEIZ unterstützt die Länder Osteuropas und der Gemeinschaft Unabhängiger Staaten (GUS) seit Anfang der neunziger Jahre in ihrem Transformationsprozess hin zu Demokratie und Marktwirtschaft. Im Jahre 2001 wendete die Schweiz für die Zusammenarbeit mit Osteuropa insgesamt rund 191 Millionen Franken auf, je rund zur Hälfte für Massnahmen der technischen und der finanziellen Zusammenarbeit. Zwei Drittel entfiel auf die Länder in Südosteuropa. Für die Bundesrepublik Jugoslawien (BRJ), d.h. Serbien, inkl. Kosovo und Montenegro, wurde ein Sonderprogramm entwickelt. Einige wichtige Dokumente der DEZA und des seco orientieren über die geleistete Arbeit und die für die mittelfristige Zukunft anvisierten Ziele.

Im März 2002 unterbreitete der Bundesrat dem Parlament den Bericht über die Zusammenarbeit des Bundes mit den Staaten Osteuropas und der GUS 2000/2001 ${ }^{1}$. Dieser stellt einen Rechenschaftsbericht über die Arbeit im Jahre 2000 und 2001 dar. Zusammen mit den ausführlichen Anhängen enthält der Bericht wichtige Aussagen über die Erfolge und auch Misserfolge bei der Umsetzung der Programme und Projekte in den Ländern Osteuropas, wo sich der Transformationsprozess jeweils sehr unterschiedlich vollzieht.

Ebenfalls im März 2002 veröffentlichte das seco seine Strategie 2006 für den Leistungsbereich Entwicklung und Transition. Die Strategie ist Vorgabe für die Zusammenarbeit mit den Entwicklungsländern und mit den Transformationsländern Osteuropas und der GUS. Sie nennt als Hauptziel des Auftrages die Verminderung der Armut, besonders auch in den Transitionsländern, wo sich der Prozess vor allem in den Ländern Südosteuropas, Zentralasiens und des Kaukasus bedeutend langsamer und langwieriger vollziehe als vorgesehen.

Der Jahresbericht über die Zusammenarbeit mit Osteuropa 2001 befasst sich speziell mit der Entwicklung in dieser Region und stellt Beispiele der Zusammenarbeit vor, wie die Lieferung von ausgedienten Basler Trams für den Strassenverkehr in Belgrad oder die Reaktivierung eines Instituts für öffentliche Verwaltung zu einem Referenzzentrum für die Unterstützung der Reformen in der BRJ.

\subsection{DIE INSTRUMENTE}

Die Zusammenarbeit mit Osteuropa basiert auf Artikel 17 des gleichnamigen Bundesbeschlusses vom 24. März 1995. Sie setzt sich zusammen aus der

Von Monika Egger, Ökonomin/Konsulentin im Bereich Wirtschaft und Entwicklung.

1 Bericht über die Zusammenarbeit des Bundes mit den Staaten Osteuropas und der GUS 2000/2001, Bern, März 2002. Interessanter Rechenschaftsbericht der DEZA und des seco ans Parlament über ihre gemeinsame Arbeit in Osteuropa und der GUS. 
Entwicklungshilfe (APD) für die Entwicklungsländer und -regionen und der öffentlichen Hilfe für die sog. Übergangsländer, die als in der Entwicklung fortgeschrittener eingestuft sind.

Die APD-Länder Osteuropas sind folgende ${ }^{2}$ :

๖ Am wenigsten entwickelte Länder: keine.

๖ Sonstige einkommensschwache Länder: Armenien, Aserbaidschan, Kirgisistan, Moldawien, Tadschikistan, Turkmenistan.

๖ Länder mit mittlerem Einkommen/untere Einkommensstufe: Albanien, Georgien, Kasachstan, Usbekistan.

๖ Länder mit mittlerem Einkommen/obere Einkommensstufe : Kroatien

๖ Länder mit hohem Einkommen: Slowenien.

Finanziert wird die Zusammenarbeit mit Osteuropa und der GUS aus dem Rahmenkredit III von 1998 (900 Millionen Franken) und seiner im Sommer 2002 vom Parlament genehmigten Aufstockung um weitere 500 Millionen Franken, verbunden mit der Verlängerung des Rahmenkredites um weitere zwei Jahre. Die Verpflichtungen werden meist für mehrjährige Projekte und Programme eingegangen. Die pro Jahr ausgewiesenen Aufwendungen stellen die im betreffenden Jahr ausbezahlten Beträge dar.

데 JSDW 2002, Tabelle 26, S. 294.

Zuständig für die Umsetzung der Ostzusammenarbeit sind die Abteilung für die Zusammenarbeit mit Osteuropa und der GUS (AZO) der DEZA (EDA) für die technische Zusammenarbeit und der Leistungsbereich Entwicklung und Transition des seco (EVD) für die finanzielle Zusammenarbeit. Die beiden Abteilungen von zwei verschiedenen Ämtern haben in den letzten Jahren ihre Zusammenarbeit verbessert und arbeiten heute weitgehend komplementär. Seit 1999 arbeiten sie gemeinsam die jeweiligen Landes- und Regionalprogramme aus. In Kraft gesetzt sind solche bereits für Mazedonien, Rumänien, Bulgarien, Bosnien und Herzegowina sowie die Russische Föderation. Für Albanien besteht eine Landesstrategie, für die Unterstützungsprogramme in Kosovo und der BRJ wurden entsprechende Konzepte verfasst. In Vorbereitung sind die Landesprogramme für den Südkaukasus, die Ukraine und für Zentralasien. Durch gemeinsame Koordinationsbüros verfügen DEZA und seco über wichtige Ressourcen vor Ort, um die Projekte und Programme umzusetzen und das Monitoring, den Evaluationsprozess und das Controlling sicherzustellen.

Die Instrumente der Zusammenarbeit sind die technische Zusammenarbeit (DEZA-Bereich) und die finanzielle Zusammenarbeit (seco-Bereich). Ihre Wirkungsweise ist analog zum Einsatz in der Zusammenarbeit mit den Entwicklungsländern des Südens; die Massnahmen werden den Bedürfnissen der Länder und Regionen angepasst. Der Einsatz der Instrumente richtet sich nach den prioritären Aktionsbereichen, wie sie in der Strategie 2006 des seco und der Strategie 2010 der DEZA sowohl für die Ostzusammenarbeit wie für die Entwicklungszusammenarbeit festgehalten sind.

JSDW 2002, S. 295.

2 Gemäss der DAC-Liste der Hilfeempfängerländer, siehe Liste am Ende des Jahrbuches. 
Die Zusammenarbeit erfolgt praktisch ausschliesslich in Form bilateraler Projekte, welche die Schweiz direkt mit den Partnern in den Empfängerländern umsetzt. Dabei stellen Nichtregierungsorganisationen in den Ländern zunehmend wichtige Partner dar; sie sind besonders von Bedeutung für die Förderung des regionalen Bewusstseins und die Bereitschaft, dass Institutionen der Zivilgesellschaft aufgebaut werden und regional und lokal Verantwortung übernehmen.

Die multilateralen Massnahmen umfassen sowohl die Beiträge der Schweiz an die internationalen Finanzinstitutionen, in denen sie Mitglied ist, als auch Kofinanzierungen von umfassenden Projekten und Programmen, welche von einem einzelnen Geldgeber nicht umgesetzt werden können:

- Europäische Bank für Wiederaufbau und Entwicklung (EBRD), an deren Aktienkapital die Schweiz mit rund 400 Millionen Franken (2,3\%) beteiligt ist. Usbekistan, Aserbaidschan, Turkmenistan, Kirgisistan, Serbien und Montenegro, die Türkei und Liechtenstein sind Mitglieder der von der Schweiz angeführten Stimmrechtsgruppe bei der EBRD.

- Als Mitglied des IWF und der Weltbank führt die Schweiz die Stimmrechtsgruppe an, deren Mitglieder mehrheitlich die gleichen Länder wie die EBRD-Stimmrechtsgruppe umfassen. Während die Türkei und Liechtenstein nicht dabei sind, kommen zusätzlich Polen und Tadschikistan dazu.

- Ferner ist die Schweiz Mitglied der G-24 (Gruppe der 24 westlichen Industrieländer für die Koordination der Zusammenarbeit mit Osteuropa), der OECD und des Europarates und beteiligt sich an gemeinsamen Aktionen.

\subsection{DIE AUFWENDUNGEN 2001}

Die Aufwendungen für die Ostzusammenarbeit des Jahres 2001 waren mit insgesamt 190,7 Millionen Franken vergleichbar hoch wie im Vorjahr mit insgesamt 188,2 Millionen Franken.

Die technische Zusammenarbeit soll Eigeninitiative und Fähigkeiten in den Reformländern mobilisieren, indem dem Empfängerland qualifizierte Expertinnen und Experten zur Verfügung gestellt werden, die wirtschaftliches, organisatorisches, wissenschaftliches, technisches oder kulturelles Fachwissen vermitteln. Die nicht rückzahlbare technische Zusammenarbeit wird partnerschaftlich gemeinsam mit dem begünstigten Land realisiert.

Die finanzielle Zusammenarbeit unterstützt die Länder in der Verbesserung der wirtschaftlichen Rahmenbedingungen als Grundlage für wirtschaftliches Wachstum und Armutsbekämpfung. Es werden finanzielle Mittel, Ausrüstung und fachliches Know-how bereitgestellt für den Ausbau sozialer und wirtschaftlicher Infrastruktur, die Förderung der Privatwirtschaft, besonders von KMU, die Stärkung des Finanzsektors und die Stabilisierung der makroökonomischen Rahmenbedingungen. Die Massnahmen des seco sind die Finanzierung von Infrastruktur, Kreditgarantien, Zahlungsbilanzhilfen und Entschuldungsmassnahmen, Handels- und Investitionsförderung. 
Tabelle 11: Auszahlungen 2001, Länder und Instrumente, in Millionen Franken

\begin{tabular}{|c|c|c|c|c|}
\hline & Technische Zusammenarbeit & $\%$ & $\begin{array}{c}\text { Finanzielle Zusammenarbeit } \\
\text { (ohne Kreditgarantien }{ }^{\text {a }} \text { ) }\end{array}$ & $\%$ \\
\hline Mitteleuropa & 0.4 & & 9.1 & 10 \\
\hline Ungarn & 0.2 & & 7.5 & \\
\hline Lettland & 0.1 & & 1.0 & \\
\hline Regional & 0.1 & & 0.6 & \\
\hline Südosteuropa & 64.6 & 65 & 50.1 & 55 \\
\hline BRJ & 12.6 & & 26.4 & \\
\hline Rumänien & 6.3 & & & \\
\hline Mazedonien & 4.5 & & 3.3 & \\
\hline Bulgarien & 6.3 & & 10.8 & \\
\hline Albanien & 5.5 & & 2.0 & \\
\hline Kroatien & 0.4 & & & \\
\hline Regional & 18.3 & & 3.9 & \\
\hline GUS & 27.8 & 26 & 15.8 & 17 \\
\hline Ukraine & 3.5 & & 0.5 & \\
\hline Kirgisistan & 6.8 & & 0.1 & \\
\hline Russland & 5.7 & & 1.8 & \\
\hline Tadschikistan & 4.0 & & 4.8 & \\
\hline Zentralasien regional & 2.5 & & 1.6 & \\
\hline Moldawien & 0.0 & & 0.2 & \\
\hline Südkaukasus & 4.5 & & & \\
\hline Georgien & 1.1 & & 0.3 & \\
\hline Aserbaidschan & 0.0 & & 1.1 & \\
\hline Regional & 0.8 & & 5.5 & \\
\hline Geografisch nicht aufgeteilt & 6.8 & 10 & 16.0 & 18 \\
\hline Total & 99.6 & 100 & 91.1 & 100 \\
\hline
\end{tabular}

Quelle: Statistische Angaben 2001, DEZA/seco.

a Hinzu kommen Zusagen für Kreditgarantien im Umfang von insgesamt 293,7 Millionen Franken (per Ende 2001).

\subsection{DIE ZUSAMMENARBEIT NACH GEOGRAFISCHEN REGIONEN}

\section{$\square$ Südosteuropa}

Die Länder Südosteuropas bilden die Schwerpunktregion der Zusammenarbeit. Mit ihrem Beitritt zum Stabilitätspakt im Juni 2000 hat die Schweiz ihr Engagement für diese Region bekräftigt. Der Pakt bedeutet für die Schweiz die Chance, ihre bilateralen Erfahrungen in regional ausgerichtete Projekte und Programme einzubringen und sich besser zu vernetzen. Bis Ende 2001 belaufen sich die schweizerischen Verpflichtungen für Projekte, die den Kriterien der regionalen Ausrichtung des Paktes entsprechen, auf insgesamt rund 70 Millionen Franken; davon werden rund 45 Millionen Franken durch das seco verwaltet und 18 Millionen Franken durch die DEZA. In den Jahren 2000 und 2001 flossen je über 6,6 Milliarden Euro in die Region Südosteuropa für deren Wiederaufbau und die soziale und wirtschaftliche Entwicklung, zu deren Mobilisierung der Pakt wesentlich beitrug.

Der politische Wandel in der BRJ im Herbst 2000 schuf die Voraussetzungen, ein substantielles Unterstützungsprogramm aufzubauen, um die Reformbemühungen zu fördern. Nicht zuletzt mit der Unterstützung der Schweiz wurde das Land vom IWF und der Weltbank als Mitglied aufgenommen und wurde zum Mitglied 
der schweizerischen Stimmrechtsgruppen. Problematisch gestaltete sich 2001 die Zusammenarbeit mit Mazedonien, wo bürgerkriegsähnliche Auseinandersetzungen ausbrachen und die Projekt- und Programmarbeit erschwerten. In Bosnien und Herzegowina wurde das Wiederaufbau- und Rückkehrprogramm durch ein mittelfristiges Zusammenarbeitsprogramm abgelöst. In Albanien ist die Umsetzung der Zusammenarbeit weiterhin sehr anspruchsvoll; die labile innenpolitische Lage und schlecht qualifiziertes und motiviertes Personal in der Verwaltung erschweren die partnerschaftliche Zusammenarbeit. In Bulgarien steht die Verbesserung der Zusammenarbeit des Staates mit den Bürgerinnen und Bürgern und die Stärkung des „ownership“ durch die Partner im Vordergrund. In Rumänien konnte die Ausrichtung der Zusammenarbeit nach den Vorgaben des Landesprogrammes erreicht werden, insbesondere konnten auch neue Projekte zur Infrastrukturfinanzierung durch das seco geplant werden.

Die speziellen Zusammenarbeitsprogramme der DEZA und des seco zur Unterstützung des Wiederaufbaus, der Wiedereingliederung von Flüchtlingen und der Stärkung der Institutionen nach den Konflikten in Bosnien und Herzegowina, Kosovo und der BRJ haben bedeutende Mehraufwendungen zu Lasten des Rahmenkredites zur Folge.

\section{$\square$ GUS-Staaten}

Zentralasien: Das seco und die DEZA sind seit mehreren Jahren in Zentralasien präsent und fördern in ihrer Zusammenarbeit besonders auch den regionalen Ansatz. Die Entwicklung in der Region wird im Bericht 2000/20013 wie folgt beschrieben: „Bis zum 11. September 2001 war die Schweiz in Zentralasien einer der wenigen bilateralen und vor Ort präsenten Geldgeber. Mit diesem Datum traten neben Afghanistan auch Kirgisistan, Tadschikistan, Usbekistan und Turkmenistan ins Rampenlicht der internationalen Gemeinschaft. ... Mit der „Entdeckung " Zentralasiens strömen sichtbar mehr Mittel und Geber in die Region, findet aber auch eine neue Dimension, nämlich Terrorismusbekämpfung, Eingang in die internationale Zusammenarbeit. Diese Situation birgt das Risiko in sich, den Blick auf Symptome statt Ursachen zu verengen, damit autoritäre Strukturen zu stützen und der Korruption Auftrieb zu verleihen (2.2 GUS-Staaten)“.

Die Schweiz ihrerseits hat ihr Engagement in Zentralasien seit längerem ausgebaut; das Schwergewicht fällt auf die zentralasiatischen Mitgliedsländer der Stimmrechtsgruppe, davon insbesondere auf die ärmsten, das heisst Kirgisistan, Tadschikistan, und teilweise auch Usbekistan. Während in Tadschikistan der Übergang von der humanitären Hilfe zur längerfristigen Zusammenarbeit vollzogen werden konnte, sind andere Länder in ihrem Transformationsprozess schon etwas weiter, wie etwa in Kirgisistan, dem ,ältesten“ Partnerland der Schweiz, wo das Programm weiter konsolidiert werden konnte. In Usbekistan ist die Zusammenarbeit erst im Aufbau. In Aserbaidschan konzentriert sich die Zusammenarbeit auf den Infrastrukturbereich (in erster Linie Wasser). Die Zusammenarbeit mit Turkmenistan wurde vor allem regional umgesetzt.

Die Zusammenarbeit mit Russland konzentriert sich auf ausgewählte Bereiche in den geografischen Regionen Perm und Nijni Novgorod. Mit der Ukraine 
erfolgt die Zusammenarbeit in einem nach wie vor schwierigen Umfeld und mit Belarus bleibt die Zusammenarbeit weiterhin sistiert. In Moldawien wurde insbesondere das Programm der humanitären Hilfe der Schweiz mit einem Schwerpunkt der Verbesserung des Zugangs zu Trinkwasser weitergeführt.

\section{$\square$ Armutsbekämpfung als transversales Thema}

Eine besondere Herausforderung für die Zusammenarbeit mit Osteuropa und der GUS stellt die zunehmende Verarmung breiter Bevölkerungskreise, vor allem auch der Mittelklasse, dar. DEZA und seco haben als ihren Hauptauftrag die Armutsbekämpfung zum Ziel. Noch sind nicht alle Bereiche der Zusammenarbeit mit Osteuropa und der GUS systematisch und explizit auf dieses Ziel hin ausgerichtet, doch lesen sich in den aktuellen Berichten und Programmen zunehmend konkrete Anstrengungen in diese Richtung.

Ein Beispiel dafür ist das Dokument New Poverty in South-Eastern Europe Issue Paper, das von der Sektion Südosteuropa der DEZA verfasst wurde und zur Klärung des Begriffs und zum besseren Verständnis der Ursachen und Dynamik der ,neuen Armut“ beitragen soll. Die neue Armut wird darin als multidimensional und multikausal definiert und Armutsbekämpfung muss demnach als ein breit verstandener Prozess gestaltet sein, der auch die enge Beziehung zu Konflikten mit einbezieht. Strategien müssen vor allem auch die Behebung der strukturellen Ursachen (Arbeitslosigkeit, Zerfall der staatlichen Pensionskassen und der Gesundheitsdienste) ermöglichen, sowie die Bedürfnisse sozial schwacher Minderheiten mit einbeziehen. Mit diesem Dokument will die Sektion den Dialog und die Auseinandersetzung innerhalb der AZO und zusammen mit den Partnern anregen. So stand die 7. Jahreskonferenz der Zusammenarbeit des Bundes mit Osteuropa und der GUS vom 5. November 2002 auch unter dem Motto: Soziale Transition in Südosteuropa: Neue Armut oder Wohlfahrt?

\section{QUELLEN}

Bundesrat, Zusatzbotschaft über die Aufstockung und Verlängerung des Rahmenkredits III für die Zusammenarbeit mit Osteuropa, 14. November 2001 (Botschaft 01.073).

Deza, Concept 2000-2005, Coopération avec l'Europe de l'Est et la CEI, Bern, März 2001.

Deza/seco, Jahresbericht 2001 Zusammenarbeit mit Osteuropa.

Deza/seco, Bericht über die Zusammenarbeit des Bundes mit den Staaten Osteuropas und der GUS 2000/ 2001, Bern, März 2002.

EDA/DDC, Stratégie pour la participation de la Direction du developpement et de la coopération DDC, Pacte de stabilité pour l'Europe du Sud-Est (2001-2003), Bern, August 2001.

SDC/Department for Co-operation with Eastern Europe and the CIS, Division for South Eastern Europe, New Poverty in South-Eastern Europe, Issue Paper, Bern, Juni 2002.

seco, Leistungsbereich Entwicklung und Transition, Strategie 2006, Bern, März 2002. 